\title{
Restoring blood flow to the lateral plantar artery after elevation of an instep flap or medialis pedis flap
}

\author{
Jonathan Velazquez-Mujica ${ }^{1}$, Francesco Amendola ${ }^{2}$, Davide Spadoni ${ }^{3}$, Hung-Chi Chen ${ }^{1,4}$ \\ ${ }^{1}$ Department of Plastic Surgery, China Medical University Hospital, Taichung, Taiwan; ${ }^{2}$ Department of Plastic and Reconstructive Surgery, \\ University of Milan, Milano; ${ }^{3}$ Department of Odontostomatological Science and Maxillo-Facial Surgery, Sapienza University of Rome, Rome, \\ Italy; ${ }^{4}$ Department of Plastic Surgery and Microsurgery, China Medical University Hospital, Taichung, Taiwan
}

The instep flap and medialis pedis flap are both originate based on the medial plantar artery. The medialis pedis flap is based from the deep branch and the instep flap is based from the superficial branch. To increase the axial rotation, it is acceptable to ligate the lateral plantar artery. However, this can partially affect the blood supply of the plantar metatarsal arch. We restored the blood flow with a vein graft between the posterior tibial artery and the ligated stump. From 2012 to 2020, 12 cases of heel reconstruction, including seven instep flaps and five medialis pedis flaps, were performed with ligation of the lateral plantar artery. The stump of the lateral plantar artery was restored with a vein graft and between the posterior tibial artery and the ligated stump. Patients were followed for 18 months. Long-term results showed the vascular restoration of the lateral plantar artery remained patent demonstrated by doppler ultrasonography. Restoring blood flow to the lateral plantar artery maintains good blood supply to the toes. If the patient in the future develops a chronic degenerative disease, with microvascular complications, bypass surgery can still be performed because of the patency of both branches.

Keywords Surgical flap / Perforator flap / Melanoma / Foot ulcer

\section{Correspondence:}

Jonathan Velazquez-Mujica

Department of Plastic Surgery, China

Medical University Hospital, 2 Yuder rd. Hospital/Dorm. North District,

Taichung 40447, Taiwan

Tel: +886-4-22052121 (ext. 4855)

Fax: +886-4-22020038

E-mail: Drjonathan.vm@gmail.com

Received: March 15, $2021 \bullet$ Revised: July 29, $2021 \bullet$ Accepted: September 2, 2021

pISSN: 2234-6163 • elSSN: 2234-6171 • https://doi.org/10.5999/aps.2021.00472 • Arch Plast Surg 2022;49:80-85

\section{INTRODUCTION}

Both the instep flap and the medialis pedis flap, along with the skin and glabrous subcutaneous tissue of the sole, provide a neurosensorial skin island and protective cover for the weight bearing area of the heel. The sole, in particular, has a fibrous septa structure and thick glabrous skin; for this reason, whenever possible, local flaps are preferable than distant flaps or skin grafts because of the functional characteristics of this region.

The medial plantar artery usually is not a component of the metatarsal arch $[1,2]$ and bifurcates just medial to the abductor hallucis muscle to form a superficial and a deep branch. The deep branch passes over the tibialis posterior tendon proximal to the navicular tubercle, providing blood supply to that area [3] upon which the medialis pedis flap is based (Fig. 1). The instep flap, also known as the medial plantar artery perforator flap, was originally reported to cover moderate size defects on the heel [4]. The medialis pedis flap has also been reported to cover defects on the heel, providing the same tissue characteristics as the instep flap but with a narrower skin island $[5,6]$. 


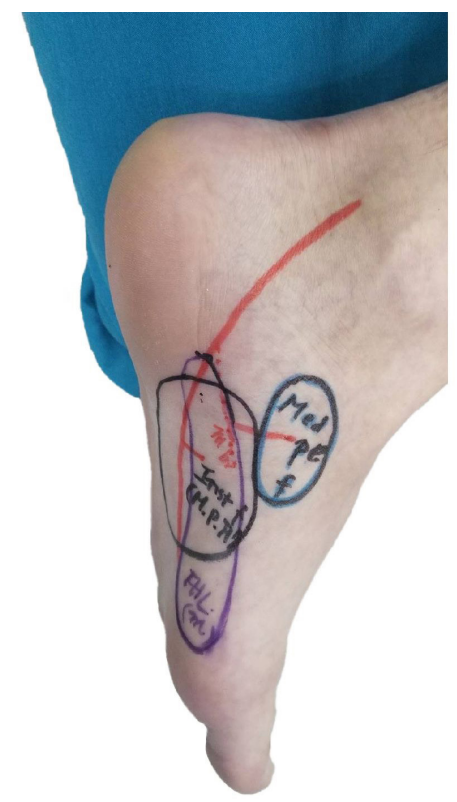

Fig. 1. Medialis pedis flap based on the deep branch of the medial plantar artery. Instep flap based on the superficial branch of the medialis pedis artery.

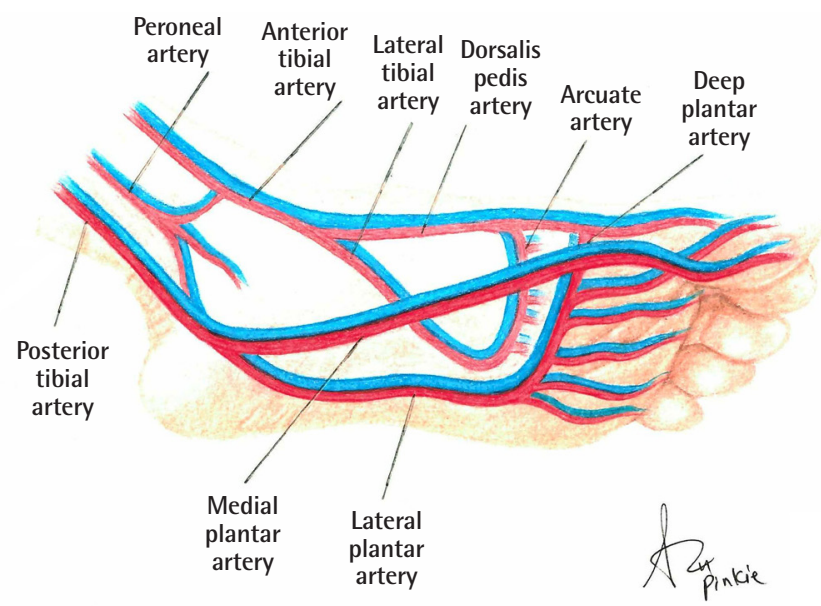

Fig. 3. Schematization of the blood supply of the foot.

In order to increase the axial rotation of the skin island to cover the entire defect on the heel, it is sometimes necessary and acceptable to ligate the lateral plantar artery (Fig. 2A) [5-7], partially affecting the blood supply of the plantar metatarsal arch by making it solely dependent on the deep plantar artery, a terminal branch of the dorsalis pedis artery (Fig. 3 ). In order to restore blood flow through the lateral plantar artery, we propose to harvest a vein graft and do perform an end to side anastomosis between the posterior tibial and the proximal stump of the previously ligated lateral plantar artery (Fig. 4).

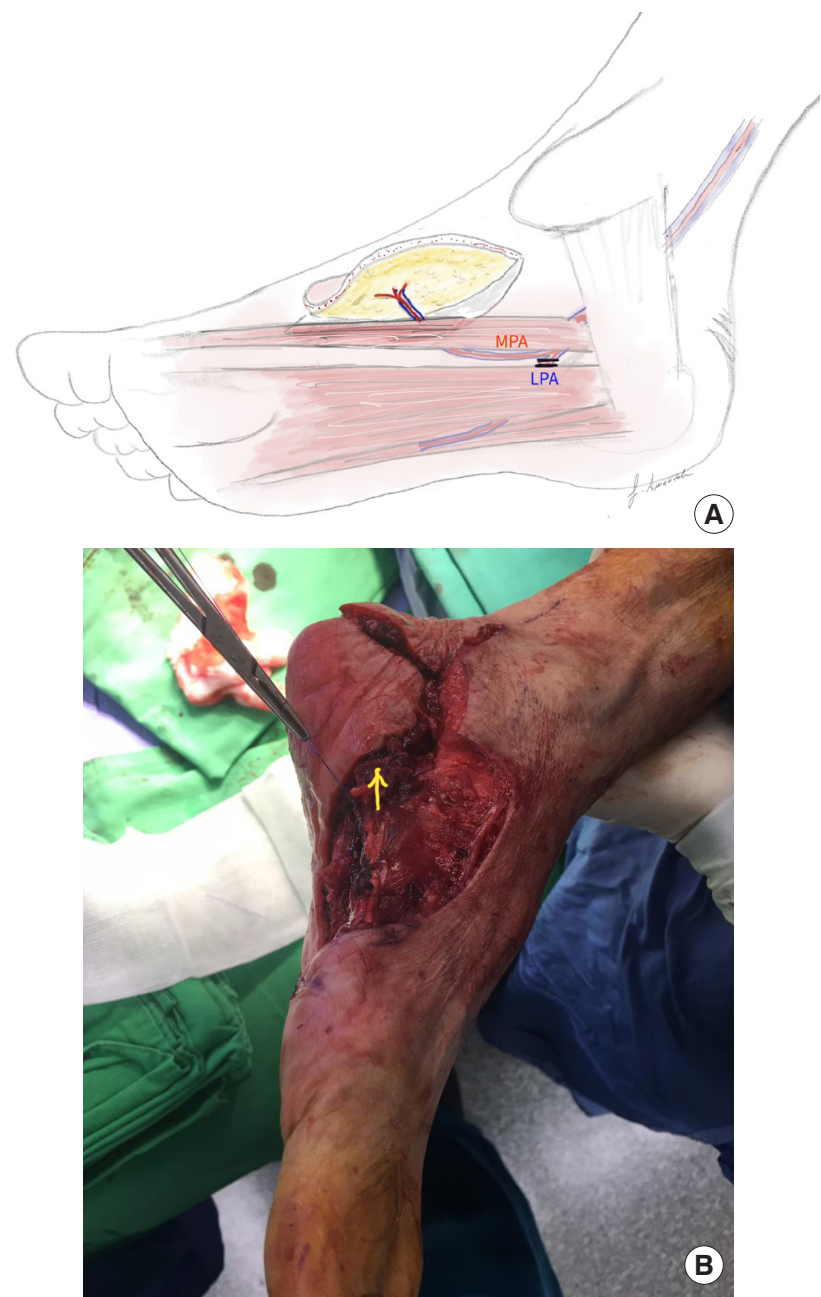

Fig. 2. Instep flap rotation. (A) Elevation of the flap. When a medialis pedis flap needs to reach a more distant location for coverage, the lateral plantar artery has to be divided. (B) Flap inset in a very distant position. The lateral plantar artery needed to be divided to reach the area. The yellow arrow shows the anastomosis to restore blood flow between the posterior tibial and the lateral plantar arteries with a vein graft. MPA, medial plantar artery; LPA, lateral plantar artery.

\section{IDEA}

\section{Methods}

This study included, 12 cases of heel reconstruction from 2012 to 2020; including seven instep flaps and five medialis pedis flaps, that where in performed ligation of the lateral plantar artery was performed in order to elongate the pedicle and cover the defect of the heel. During these procedures, the stump of the previously ligated lateral plantar artery was restored with a vein graft and an end to side anastomosis between the stump of the lateral plantar artery and the side of the posterior tibial artery. The age range of the patients was 39 to 85 years, with most in their 40's and 50's. Four patients presented with melanoma and were reconstructed with instep flaps. Eight patients present- 


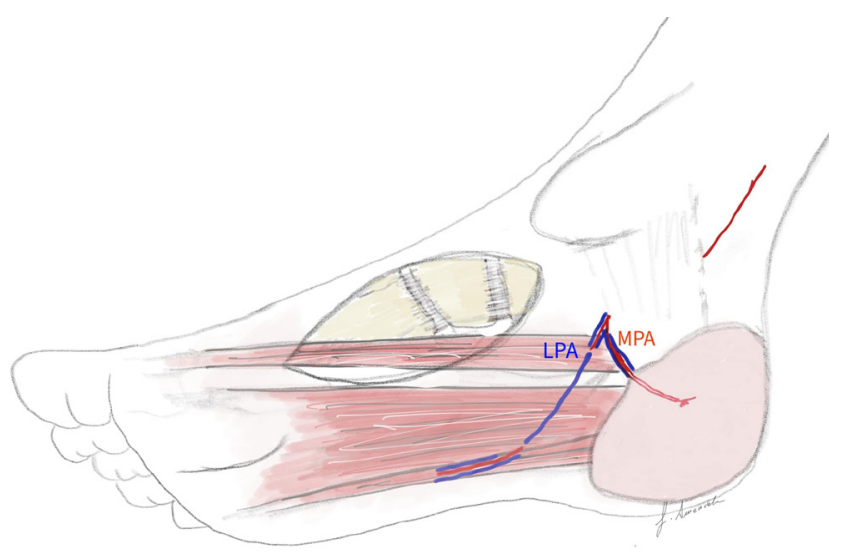

Fig. 4. Restoration of the lateral branch with vein graft. Medialis pedis flap with restoration of blood flow between the posterior tibial artery and the lateral plantar artery with vein graft (schematization). MPA, medial plantar artery; LPA, lateral plantar artery.

ed with chronic ulcers, three of them were reconstructed with instep flaps and five of them with medialis pedis flaps.

The vein graft was used to restore the gap between the tibialis posterior artery and the lateral plantar artery after its ligation was taken from the lesser saphenous vein. The gaps usually were between 6 and $8 \mathrm{~cm}$, but the vein grafts taken were usually $8-10$ $\mathrm{cm}$ to avoid tension on the anastomosis.

In all cases, surgery was performed after the informed consent was signed by the patients. This study was conducted with approval from the hospital ethics committee (IRB No. CMUH109REC3-170).

\section{Surgical technique}

The instep flap, also known as a medial plantar artery perforator flap, and the medialis pedis flap are both based originate from on the medial plantar artery; with the medialis pedis flap originating from is based on the deep branch and the instep flap from is based on the superficial branch of the medial plantar artery and its perforators (Fig. 1). The posterior tibial artery bifurcates between the medial malleolus and the heel forming the medial plantar artery and the lateral plantar artery. The medial branch of the medial plantar artery passes beneath the abductor hallucis muscle where it bifurcates into the superficial branch and the deep branch. It is situated between the flexor digitorum brevis and the abductor hallucis, at the base of the first metatarsal bone and, passes along the medial border of the big toe, anastomosing with the first dorsal metatarsal artery.

The lateral plantar artery runs distally to the base of the fifth metatarsal bone, turns medially, and unites with the deep plantar branch of the dorsalis pedis artery completing the plantar branch.

Both arteries are accompanied by the medial plantar nerve and

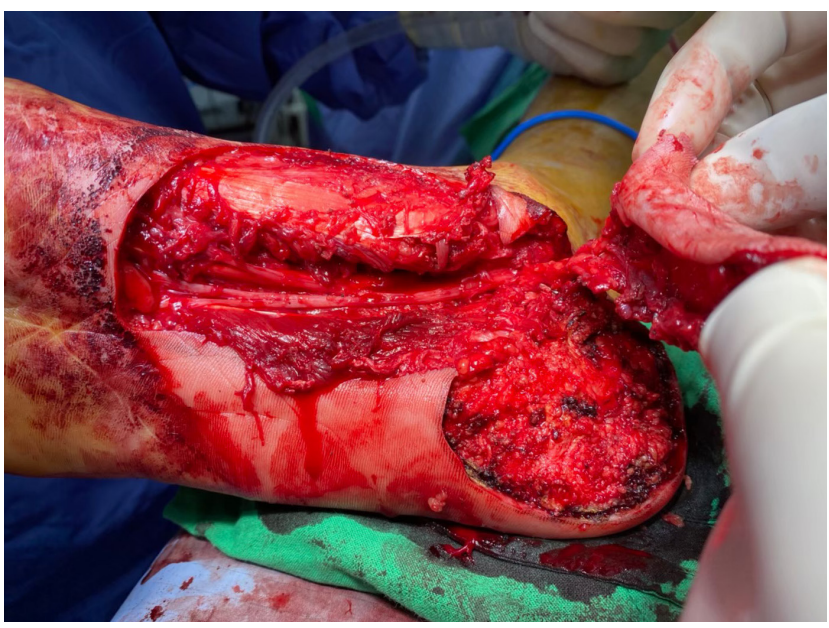

Fig. 5. Insetting of the flap. The flap is turned to cover the defect.

lateral plantar nerve and concomitant veins.

Division of the branches of the medial plantar nerve to the toe, this can cause postoperative sensory disturbances in the great toe $[7,8]$. The deep venous return from the sole is provided by venae comitantes, a superficial system that drains via the lesser and great saphenous veins.

The size of the medialis pedis flap was as large as $9 \times 6 \mathrm{~cm}$, from the medial malleolus proximally to the distal edge until at the lateral side of the middle of the first metatarsus. The instep flap was as large as $11 \times 8 \mathrm{~cm}$. In both flaps the plane of dissection was subfascial and the muscle branches had to be ligated. In order to increase the axial rotation of the flap as much as possible and avoid tension on the pedicle, the lateral plantar artery was ligated. The donor site was covered with a split-thickness skin graft.

To ligate of the lateral branch (Figs. 2A, 5) and restore its vascularity, the vein graft was taken from the lesser saphenous vein and, once the flap was transposed to cover the defect (Figs. 5, 6), the vein graft was used as a bridge between the posterior tibial artery and the proximal stump of the lateral plantar artery (Figs. 4,7 ), with end-to-side anastomosis between the posterior tibial artery and vein graft and end-to-end anastomosis between the vein graft and the proximal stump of the lateral branch of the medial plantar artery.

\section{Results}

Patients were followed for a period of 18 months. The longterm results showed that, the flaps provided good coverage when local tissue from the sole was used and patients reported sensorial discrimination to fine touch on the flaps and with weight bearing on the heel. Normal ambulation was restored in all patients. Two flaps were sutured too tight and caused mild 


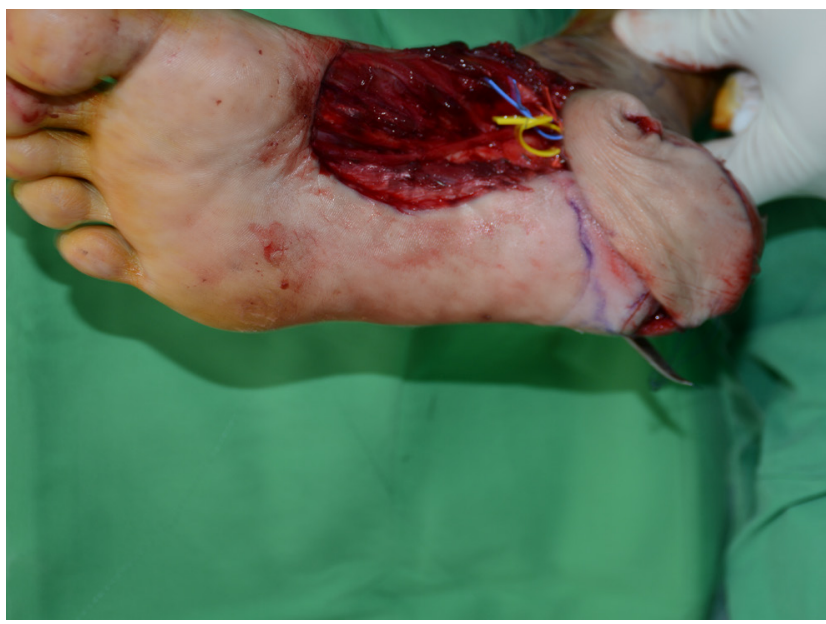

Fig. 6. Restoration of the blood flow with a vein graft. The yellow vessel loop shows the restoration of the flow of the lateral plantar artery after the vein graft.

congestion distally; these cases were resolved by removing some sutures. Two patients reconstructed with the medial plantar artery perforator flap reported sensory disturbances on the lateral side of the great toe. The vascular restoration of the lateral plantar artery remained patent, as demonstrated by Doppler ultrasonography. The skin grafts over the donor sites were fully integrated without any complications.

\section{DISCUSSION}

In order to increase axial rotation and allow the skin island to fully reach distant areas of heel defects (Fig. 2B), many surgeons have accepted that ligation of the lateral plantar artery is necessary $[2,3,5,7]$ without considering that the lateral plantar artery also completes the deep plantar arch when it anastomoses with the deep plantar branch of the dorsalis pedis artery. Therefore, the following questions arise: why has it been considered acceptable to ligate the lateral plantar artery and what is the importance of restoring the blood flow through it? These two questions can be answered by considering the anatomy of the pedal blood flow. First, look considering at the pedal arches; the superficial and deep arches communicate through the three arterial systems supplying the foot: (1) the anterior tibial artery and its branch the dorsalis pedis artery to deep plantar artery; (2) the peroneal artery with a communicating branch to the posterior tibial artery and an anterior branch anastomosed with the distal anterior tibial artery; and (3) the posterior tibial artery with the lateral plantar artery (Fig. 3) [2,3,9]. Although the plantar arch consists of the deep plantar artery (from the dorsalis pedis artery) and the lateral plantar artery (from the posterior tibial artery), the flow is also helped by the peroneal artery. That

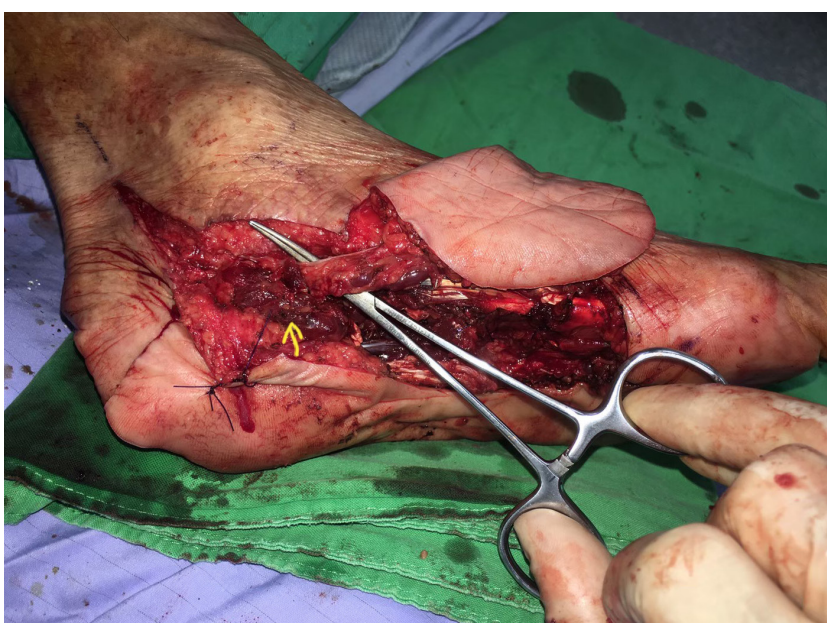

Fig. 7. Ligation of the lateral branch. The yellow arrows mark the ligated lateral plantar artery, once the flap has been elevated.

is why ligation of the lateral plantar artery has been considered acceptable in order to increase the rotation axis of the flap.

In a young patient or patient without chronic and degenerative disease, the foot circulation will not be affected immediately. Nevertheless, the effect may well be important in the future if another vascular intervention to that foot is needed, and which is answers the question of why it is important to restore the blood flow to the lateral plantar artery after its ligation. Although the flap and the anastomosis with a vein graft become stable in 14 days as a result of platelet aggregation, fibrin formation, and pseudo-intima development, and the anastomotic site and the vein graft are covered by endothelium [10], it is recommended that patients do not step on the flap for at least 1 month.

It is well known that hypertension induces physiological and morphological changes in the arterial wall. Changes in all vessel layers, from the endothelium to the perivascular adipose tissue, have been described [11] due to the repeated secretion of procontractile factors and decreased secretion of prorelaxant factors, resulting in increased the resistance of the small vessels $[12,13]$.

The vascular flow of patients with diabetes can be affected in the early and long-term stages of the disease. Macro- and microcirculatory disorders manifest because of insufficient blood flow velocity and volume in the early stages of diabetes. However, blood flow velocity is faster in patients with advanced disease and microvascular complications [14-16], including vascular tone disruption occur. Peripheral limb blood flow decreases dramatically due to vessel damage caused by hyperglycemia [17] and those patients are vulnerable to vascular endothelial dysfunction.

Atherosclerotic disease below the knee is a risk shared by pa- 
tients with diabetes mellitus and peripheral vascular disease without diabetes. This can also lead to decreased volume perfusion in the lower limb [18] and has been traditionally treated with bypass surgery. The bypass inflow originates in different locations such as the superficial femoral artery and the popliteal artery, above and below the knee. The recipient vessels dorsalis pedis artery, the tarsal artery, and the medial or lateral plantar arteries in patients with critical limb ischemia. When compared to endovascular treatments like percutaneous transluminal angioplasty, bypass surgery has been considered the more effective type of revascularization [19].

Bypass to the medial or lateral plantar artery or to the lateral tarsal artery is less frequently performed and were as, noted to have a $15 \%-29 \%$ rate of early graft occlusion [20].

In conclusion, for patients who may require revascularization of the lower limb at some point in the future, restoring blood flow to the lateral plantar artery will preserve the option to use this artery as a recipient in bypass surgery.

\section{NOTES}

\section{Conflict of interest}

No potential conflict of interest relevant to this article was reported.

\section{Ethical approval}

The study was approved by the Research Ethics Committee of the China Medical University Hospital (IRB No. CMUH109REC3-170) and performed in accordance with the principles of the Declaration of Helsinki. Written informed consent was obtained.

\section{Patient consent}

The patients provided written informed consent for the publication and the use of their images.

\section{Author contribution}

Conceptualization: J Velazquez-Mujica, HC Chen. Data curation: HC Chen. Formal analysis: J Velazquez-Mujica, HC Chen. Methodology: J Velazquez-Mujica, D Spadoni. Project administration: J Velazquez-Mujica, HC Chen. Visualization: J Velazquez-Mujica, F Amendola, D Spadoni. Writing - original draft: J Velazquez-Mujica. Writing - review \& editing: J Velazquez-Mujica, D Spadoni, H Chen.

\section{ORCID}

Jonathan Velazquez-Mujica

https://orcid.org/0000-0001-5909-5970
Francesco Amendola https://orcid.org/0000-0002-1410-7568

Davide Spadoni https://orcid.org/0000-0003-1223-1458

Hung-Chi Chen https://orcid.org/0000-0002-2749-1909

\section{REFERENCES}

1. Snyder GB, Edgerton MT Jr. The principle of the island neurovascular flap in the management of ulcerated anesthetic weightbearing areas of the lower extremity. Plast Reconstr Surg 1965;36:518-28.

2. Rodriguez-Vegas M. Medialis pedis flap in the reconstruction of palmar skin defects of the digits: clarifying the anatomy of the medial plantar artery. Ann Plast Surg 2014;72: 542-52.

3. Masquelet AC, Romana MC. The medialis pedis flap: a new fasciocutaneous flap. Plast Reconstr Surg 1990;85:765-72.

4. Harrison DH, Morgan BD. The instep island flap to resurface plantar defects. Br J Plast Surg 1981;34:315-8.

5. Kim SW, Hong JP, Chung YK, et al. Sensate sole-to-sole reconstruction using the combined medial plantar and medialis pedis free flap. Ann Plast Surg 2001;47:461-4.

6. Park JS, Lee JH, Lee JS, et al. Medialis pedis flap for reconstruction of weight bearing heel. Microsurgery 2017;37: $780-5$.

7. Miyamoto Y, Ikuta Y, Shigeki S, et al. Current concepts of instep island flap. Ann Plast Surg 1987;19:97-102.

8. Trevatt AE, Filobbos G, Ul Haq A, et al. Long-term sensation in the medial plantar flap: a two-centre study. Foot Ankle Surg 2014;20:166-9.

9. Basit H, Eovaldi BJ, Sharma S. Anatomy, bony pelvis and lower limb, peroneal artery. In: StatPearls [Internet]. Treasure Island: StatPearls publishing; 2021 Jan [cited 2021 Mar 14]. Available from: https://www.ncbi.nlm.nih.gov/books/ NBK538280/.

10. Raine T. Microvascular techniques. In: Jurkiewicz MJ, Krizek TJ, Mathes SJ, et al., editors. Plastic surgery: principles and practice. St. Louis: Mosby; 1990. p. 1573-91.

11. Martinez-Quinones P, McCarthy CG, Watts SW, et al. Hypertension induced morphological and physiological changes in cells of the arterial wall. Am J Hypertens 2018;31:106778.

12. Conway J. A vascular abnormality in hypertension. A study of blood flow in the forearm. Circulation 1963;27(4 Pt 1): 520-9.

13. Cade WT. Diabetes-related microvascular and macrovascular diseases in the physical therapy setting. Phys Ther 2008; 88:1322-35.

14. An Y, Kang Y, Lee J, et al. Blood flow characteristics of dia- 
betic patients with complications detected by optical measurement. Biomed Eng Online 2018;17:25.

15. Williams DT, Price P, Harding KG. The influence of diabetes and lower limb arterial disease on cutaneous foot perfusion. J Vasc Surg 2006;44:770-5.

16. Fowler MJ. Microvascular and macrovascular complications of diabetes. Clin Diabetes 2008;26:77-82.

17. Williams DT, Pugh ND, Coleman DP, et al. The laboratory evaluation of lower limb perfusion in diabetes mellitus: a clinical review. Br J Diabetes Vasc Dis 2005;5:64-70.
18. Lowry D, Saeed M, Narendran P, et al. A review of distribution of atherosclerosis in the lower limb arteries of patients with diabetes mellitus and peripheral vascular disease. Vasc Endovascular Surg 2018;52:535-42.

19. Houlind K. Surgical revascularization and reconstruction procedures in diabetic foot ulceration. Diabetes Metab Res Rev 2020;36 Suppl 1:e3256.

20. Ascer E, Veith FJ, Gupta SK. Bypasses to plantar arteries and other tibial branches: an extended approach to limb salvage. J Vasc Surg 1988;8:434-41. 